\title{
Estructura de los relatos sobre experiencias personales en hablantes del otomí de Santiago Mexquititlán
}

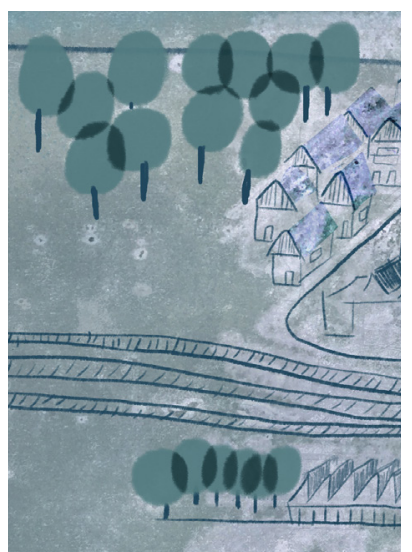

Karola Rico

Universidad Autónoma de Querétaro, México

jkarolarico@gmail.com

Eva Patricia Velásquez Upegui

Universidad Autónoma de Querétaro, México

evapvelasquez@gmail.com

\section{Resumen}

En este artículo se analizan 28 textos sobre experiencias personales de hablantes urbanos de otomí-español. Se describen las tendencias estructurales y los componentes de estos textos con base en la propuesta de Labov (1972) para observar si sus características tienen correspondencia con la estructura de las narraciones en lenguas ya descritas. El corpus pertenece a la variante del otomí de Santiago Mexquititlán, producido por cinco hombres y cinco mujeres con edades entre 45 y 65 años. Estos colaboradores migraron de su pueblo de origen y han estado viviendo en la ciudad de Querétaro desde hace 20 años. Los textos hacen referencia a dos tópicos: un día de juego en la infancia y el primer día en la ciudad. Se encuentra que los textos con referencia al primer tópico se estructuran como guiones (Schank y Abelson 1977), mientras que los textos sobre el primer día en la ciudad se presentan como narraciones. Estas últimas contienen como mínimos estructurales la evaluación, la orientación y la acción complicante. Los guiones, por su parte, cumplen con las condiciones de entrada, los accesorios y los roles. Por tanto, los textos revisados comparten las características estructurales encontradas en otras lenguas con una distribución particular en otomí.

\section{Structure of stories about personal experiences in speakers of the Otomí of Santiago Mexquititlán}
Abstract
This research paper analyzes, from a discursive approach, 28 texts relating to personal experiences of bilingual speakers of Otomi-Spanish. We descri- be the components of the texts based on the proposal of Labov (1972) in

Palabras clave

experiencias personales superestructura narración guiones cláusulas otomí

Keywords

personal narratives superstructure narratives scripts

clauses Otomi 
order to identify if their characteristics correspond to the structure of the narratives in languages already described. The data analyzed belongs to the Otomi variant of Santiago Mexquititlán, Amealco. The corpus is based on interviews with speakers; five women and five men, between 45 and 65 years of age, with Otomi as L1 and Spanish as L2. These collaborators migrated from their originating village and they have been living in the city of Querétaro for approximately 20 years. The texts refer to two cultural topics; games played during their childhood and their first day in the city. The data shows that the structure of the texts relating to the first topic tend to be scripts (Schank and Abelson 1977), while the texts, with regard to their first day in the city, have a tendency to be personal narratives. Of the six components that Labov (1972) proposes, the latter presents at a minimum 'evaluation', 'orientation' and 'complication'. Finally, the scripts achieve the 'entry conditions, the 'props' and 'roles'; therefore, the revised texts share the structural characteristics found in other languages with a particular distribution in Otomi.

\section{Estrutura de histórias sobre experiências pessoais em falantes do otomí de Santiago Mexquititlán}

\section{Resumo}

Neste artigo, 28 textos sobre experiências pessoais de falantes urbanos de otomi-espanhol são analisados. As tendências estruturais e os componentes destes textos são descritos com base na proposta de Labov (1972), para observar se as características deles têm correspondência com a estrutura das narrações de outras línguas já descritas. O corpus pertence à variedade do otomi de Santiago Mexquititlán (México), e é produzido por cinco homens e cinco mulheres de 45 a 65 anos que migraram da aldeia de origem deles e têm estado morando 20 anos na cidade de Querétaro. Os textos falam sobre duas matérias: um dia de jogo na infância e o primeiro dia na cidade. Segundo os resultados, os primeiros textos são estruturados como roteiro, enquanto os segundos são aprensentados como narrações; estas apresentam como mínimos estruturais a avaliação, a orientação e a acção complicante, enquanto os roteiros cumprem as condições de entrada, os accesórios e os papéis. Portanto, os textos estudados compartilham as características estruturais já registradas em outras línguas, mas apresentam uma distribuição especial em otomi.

\section{Introducción}

El discurso narrativo está presente en la mayoría de los contextos de la interacción social y es parte integral de la manera como estructuramos, damos cuenta y exponemos el entendimiento de nuestra condición y experiencia humana (Thornborrow 2012). A través de este discurso, los pueblos preservan su identidad y memoria para transmitir intergeneracionalmente sus saberes. En las lenguas originarias, las narraciones de experiencias personales han sido poco documentadas y, por ende, poco descritas desde un enfoque discursivo. En este sentido, el objetivo de este documento es describir cómo los hablantes otomíes ${ }^{1}$ representan estructuralmente la percepción de los eventos en estos relatos y cuál es la información que privilegian al momento de contar sus experiencias personales. Para ello se analiza la superestructura de las historias

\section{Palavras-chave}

experiências pessoais superestruturas narração roteiro cláusula otomi
1. Las lenguas otomíes pertenecen al grupo oto-pameano de la familia lingüística oto-mangue conformada por los grupos tlapaneco-mangue, popoloca-zapoteco, amuzgo miztecano, otopame y chinanteco. Constituyen el grupo otopame dentro de la rama otomiana (otomí, mazahua, matlatzinca y ocuilteco) que lo integra junto con la pameana (pame y chichimeca). De acuerdo con la información del Catálogo de lenguas indígenas consignadas por el Instituto Nacional de Lenguas Indígenas (INALI) para el 2008, como agrupación lingüística, el otomí está compuesta por las siguientes nueve variantes lingüísticas: 1) otomí de la Sierra, 2) otomí bajo del noroeste, 3) otomí del oeste, 4) otomí del oeste del Valle del Mezquital, 5) otomí del Valle del Mezquital, 6) otomí de Ixtenco, 7) otomí de Tilapa o del sur, 8) otomí del noroeste, y 9) otomí del centro (Nava López 2010). 
narradas por los hablantes bilingües de otomí-español, se describen los segmentos narrativos y se analizan los componentes estructurales de estos relatos.

En este artículo se presenta, en primer lugar, el concepto de superestructura textual. Enseguida se hace una revisión teórica sobre las características y componentes de las narraciones de experiencias personales y se explican los segmentos narrativos que se presentan en estos relatos. Posteriormente se describen las características de los narradores y la metodología llevada a cabo para la recolección del corpus, su organización y descripción. Luego se continúa con el análisis y la discusión de los resultados para terminar con los comentarios finales en los que se recapitulan los hallazgos encontrados.

\section{Marco teórico}

\subsection{La narración}

El discurso narrativo ha sido ampliamente definido desde diferentes perspectivas. Principalmente, se ha considerado la relación temporal de los eventos y la intencionalidad del participante para resolver una situación problemática (Ochs 1992, Bruner 1986, Nelson 1996). Desde el punto de vista discursivo, Bamberg (1997) define la narración como una construcción de unidades temporales guiadas por la actividad discursiva, mientras que Stein y Albro (1997) señalan que es todo texto organizado causalmente y dirigido hacia una meta. La narración también se ha considerado como una construcción social y cultural que depende del contexto narrador (Nicolopoulou 1997, Quasthoff 1997, McCabe 1997).

De acuerdo con Labov y Waletzky (1997 [1967]), la narración es cualquier secuencia de cláusulas que contiene al menos una juntura temporal, es decir, es un método de recapitulación de experiencias pasadas que hace coincidencias entre una secuencia verbal de cláusulas y una secuencia de eventos que realmente ocurrieron. En este sentido, entendemos la narración como un discurso que posee dos rasgos inherentes, la ruptura de la cotidianeidad y la secuencia temporal, enmarcados en un contexto sociocultural que contribuye con la construcción del relato.

\subsection{Características de las narraciones: superestructura}

Van Dijk (1980) señala que el contenido de los textos en el plano global se organiza en dos tipos de estructuras: la macroestructura y la superestructura textual. La primera se refiere a la estructura semántica del conjunto del texto y la segunda, a la forma en la que la información del texto se organiza, es decir, la estructura textual formal. Las frases de un texto se organizan en un esquema constituido por categorías funcionales. Algunas de estas son obligatorias y otras son opcionales.

En el trabajo pionero sobre la estructura de la narrativa oral de Labov y Waletzky (1967) y Labov (1972), los autores encontraron que, en las historias recolectadas por medio de entrevistas sociolingüísticas, emerge una sintaxis identificable para el discurso narrativo, un modelo estructural que de una u otra manera ha proporcionado las bases para gran parte del análisis narrativo en las últimas cuatro décadas. 
Labov (1972) propone que hay componentes que habitualmente están presentes en la narración oral e identifica seis rasgos de una narrativa completa: 1) un resumen (opcional), que puede proveer una síntesis de la historia que se contará; 2) una evaluación, que señala el valor o punto de la historia, lo que hace que sea digna de ser contada (Thornborrow y Coates 2005), es decir, el medio utilizado por el narrador para indicar el punto de una narrativa; 3) la orientación (el quién, dónde y cuándo), los segmentos de la escena situada que ubican la narración en cuanto a tiempo, lugar, personas, situación; 4) una complicación, las cláusulas que forman la trama de una narración; 5) la resolución de esta última, es decir, la terminación o resultado de la acción que complica; y 6) la coda, que como el resumen es opcional, y es la parte de la historia que señala el final de la narración y que forma un puente entre el tiempo de la historia y el tiempo presente de la conversación.

Estos elementos estructurales desarrollados por Labov, también los incluye Van Dijk (1980), quien señala que la primera categoría típica de las narrativas es el escenario, que generalmente caracteriza descripciones de la situación original, presenta los participantes, el tiempo y el lugar donde se llevan a cabo los episodios. En una narración, después de ubicar estos elementos, se da cuenta del "¿qué pasó en ese escenario?". Esta categoría es llamada complicación y se trata de una acción que es digna de ser contada y que típicamente es un evento peligroso, divertido o simplemente inesperado. En general, la complicación requiere la categoría de resolución o la solución que se da al evento dificultador. Se trata de una (re-)acción de una persona a un evento o acción previa. Según Van Dijk, la evaluación es otro aspecto importante en las narrativas, dado que caracteriza la reacción emocional o mental del participante o el narrador con respecto al episodio narrado. Por último, la coda o moral, que señala una conclusión de los eventos, no se trata solo de una categoría única de las fábulas o parábolas, sino también de historias sobre experiencias personales, especialmente cuando son enunciadas con el propósito de informar a alguien sobre las posibles consecuencias de una determinada acción.

\subsection{Estudios sobre la narración en otomí}

Los estudios sobre las narraciones en otomí aún son escasos. No obstante, Bartholomew (1995) hace un análisis sobre los tipos de difrasismos y sus funciones en el discurso narrativo del otomí del Estado de México. Además, señala que estos hacen resaltar el hecho que se describe y tienen una fuerte tendencia a yuxtaponer dos oraciones independientes en la construcción de un periodo complejo. La autora muestra que hay un índice muy alto de difrasismos en la narración otomí. Los tipos de difrasismos incluyen la repetición exacta de la primera oración, la variación sobre el tema de la primera, la adición de otros detalles y hasta otra expresión de la misma idea.

Bartholomew (1984), en otro estudio, presenta una discusión sobre las diferencias de estilo entre el discurso narrativo oral y escrito. Hace énfasis en que estas diferencias son más evidentes cuando el relato oral se hace de manera espontánea. En esos casos, la versión oral tiende a tener una organización poco integrada en la que quien narra relata los eventos tal como le vienen a la mente. La anécdota escrita, en cambio, suele organizar los eventos en oraciones complejas y hace uso de la subordinación. Sin embargo, no es siempre absoluta la diferencia entre ambas modalidades del discurso. 
Palancar (2009a), en su gramática del otomí de San Ildefonso Tultepec, hace algunas notas con respecto a la narración. Para ejemplificar los estilos narrativos, presenta un discurso de naturaleza oral. Se trata de un extracto de 22 cláusulas $^{2}$ en el que se hace evidente la elisión de marcadores de Tiempo, Aspecto y Modo (TAM), que son recuperables en contexto. Otra de las características de este texto es la intercalación de tiempos y modos en la narración, alternando entre el realis y el irrealis. Además, Palancar encuentra que existe una alta presencia de verbos dicendi en tiempo presente, tanto en discurso directo como indirecto, y explica que el tiempo de estos verbos produce el efecto narrativo en el que lo dicho se acerca al presente del acto del habla desde donde se cuenta la historia. De acuerdo con el autor, los eventos que se llevan a cabo secuencialmente en la vida real se codifican a través de una secuencia yuxtapuesta de cláusulas paratácticas, sin ningún conector discursivo.

Sobre el foco narrativo, Palancar (2009a) agrega que, cuando una frase nominal presenta una información de sorpresa que se supone relevante para la recepción de la narrativa, se introduce con el adverbio hont'ä, que en español se traduce como "solamente". De esta forma, se marca el foco narrativo. Este constituyente siempre se emplaza en una posición preverbal $y$, si la frase nominal funciona como sujeto, se posiciona obligatoriamente como sujeto topical.

Castillo (2014) toma como referencia las aportaciones de Palancar (2009a) y analiza, en términos del modelo de la Estructura Argumental Preferida, ejemplos de narrativas en cora, zapoteco y otomí. En su investigación, describe la distribución de estructuras focales en cada narrativa y propone una clasificación de las tres lenguas siguiendo la propuesta de Van Valin (1999). Castillo (2014) toma los datos del cuento de Palancar (2009b) titulado Nor Kamalio "El Camaleón”, una narración folclórica de la región de Amealco constituida por 127 cláusulas. De acuerdo con la autora, 70 de ellas son intransitivas. En su análisis, señala que en otomí parece no haber restricciones en la posición de los argumentos focalizados ni en el orden de constituyentes, de modo que el otomí se puede clasificar como una lengua de pragmática flexible.

\subsection{Metodología}

\subsubsection{Narradores}

Los datos se recopilaron a partir de entrevistas a hablantes bilingües que tienen el otomí como lengua materna y que aprendieron el español como segunda lengua. Todos los narradores son originarios de Santiago Mexquititlán, Amealco ${ }^{3}$. Se entrevistaron cinco hombres y cinco mujeres de la generación de abuelos, adultos maduros y mayores ${ }^{4}$ con edades entre 45 y 65 años, los cuales llevan 20 años viviendo en la Colonia Nueva Realidad, al sureste de la Ciudad de Querétaro.

Se decidió entrevistar a esta generación de hablantes debido a que hablan con mayor fluidez su lengua originaria. De acuerdo con Rico (2014), las generaciones más jóvenes de habitantes otomíes de la colonia Nueva Realidad ya no hablan el otomí. Además, se realizó una prueba piloto en la que se entrevistó a hablantes con un rango de edad de entre 24 y 65 años y se encontró con que los hablantes menores de 45 años contaron sus historias mayormente en español con solo algunos términos en otomí.
2. Palancar (2009a), en su Gramática y textos del hñöñhö, ofrece un compendio de 12 textos en la lengua originaria con traducción literal al español. El extracto con el que el autor explica los estilos narrativos del hñöñhö se corresponde con las primeras 22 cláusulas del texto 3, cuya versión completa cuenta con 183 cláusulas.
3. Se realizó una exploración sobre el uso del otomí y el español con base en el cuestionario sobre el uso de idiomas elaborado en Brain, Language and Computation Lab de Penn State University (Li et al. 2014). Este instrumento revisa el uso de las lenguas con diferentes interlocutores (cónyuge, hijos, nietos, amigos, madre, padre, hermanos) y el de la L1 y la L2 en distintas actividades (cálculos aritméticos, recordar números, soñar, pensar, hablar solo, y expresar emociones). Se puntúa la frecuencia de uso en una escala de valores del 1 al 5 , donde 1 corresponde a 'nunca', 2 es 'a veces', 3 es 'frecuentemente', 4 es 'muy frecuentemente', y s es 'siempre'. Se encontró que los narradores de este estudio son hablantes bilingües cuya lengua materna es el otomí y la utilizan con mayor frecuencia con todos los interlocutores, además de utilizar de manera equilibrada las dos lenguas en las actividades antes mencionadas.

4. Donati (1999) propone hablar de generación desde la perspectiva de la sociología relacional. Así, generación es el conjunto de personas que comparten una relación, aquella que liga su colocación en la descendencia propia de la esfera familiar-parental (esto es: hijo, padre, abuelo, etc.) con la posición definida en la esfera societal con base en la "edad social" (es decir: de acuerdo con los grupos de edad: jóvenes, adultos, ancianos, etc.). 


\subsubsection{Instrumentos}

Para la obtención de los relatos, se realizó una entrevista en la que se les pidió a los narradores que contaran la historia de su primer día en la ciudad y la de un día de juego que recordaran de su infancia. Se eligieron estos tópicos, pues son temas que la misma comunidad de estudio considera culturalmente pertinentes. Esto se determinó a partir de una investigación realizada por Rico (2014), cuyo objetivo era diseñar una propuesta didáctica para la enseñanza-aprendizaje intergeneracional del otomí en la comunidad urbana de migrantes procedentes de Santiago Mexquititlán, Querétaro. Se llevaron a cabo talleres con padres y abuelos, en los que se decidieron los temas culturales de mayor relevancia para socializar con los niños. Entre ellos se incluyeron los temas que hacen parte de la presente investigación. Estos dos temas permiten, además, observar si la estructura textual varía o se mantiene con independencia del evento narrado.

Los datos fueron audiograbados tanto en otomí como en español durante la misma sesión. Los hablantes produjeron los textos inicialmente en la lengua originaria otomí y después en español. En total se grabaron 28 textos en cada versión. Todos los relatos fueron colectados a través de entrevistas sociolingüísticas en el periodo de enero a marzo de 2017.

\subsubsection{Tratamiento de la información}

Se transcribieron los datos y se agregó una traducción libre de las historias narradas en lengua originaria con la colaboración de Pascual Lucas Julián ${ }^{5}$, hablante de otomí de la misma comunidad.

Se realizó la transcripción total de los datos en el procesador de textos Microsoft Word y una transcripción parcial en el software Praat (Boersma y Weenink 1992) para aclarar algunas dudas fonéticas que se tenían sobre segmentos del discurso. La ortografía utilizada en la transcripción corresponde con la propuesta de Hekking et al. (2010). Los datos se glosaron con base en Leipzig Glossing Rules (Comrie y Haspelmath 2015) y las propuestas de Hekking y Andrés de Jesús (1984) y Palancar (2009a) ${ }^{6}$.

Los textos se segmentaron por cláusulas, siguiendo a Berman y Slobin (1994), quienes proponen hacer uso de la cláusula como unidad de transcripción y codificación del discurso para el análisis lingüístico ${ }^{7}$. Finalmente, se clasificaron los 28 textos de acuerdo con sus características estructurales a partir de las propuestas de Van Dijk (1980), Labov y Waletzky (1967), Labov $(1972)^{8}$, y Schank y Abelson (1977).

\subsubsection{Análisis y resultados}

Los criterios para la clasificación de los textos se basan en sus componentes estructurales y en la naturaleza de los eventos. Para clasificar un texto como narración, se requiere, entonces, que se trate de un evento único en el pasado, es decir, una situación que rompe con lo cotidiano y es digna de ser contada. Para Labov (1972), los mínimos estructurales en una narración consisten en dos cláusulas marcadas temporalmente como pasado - las que constituyen una secuencia narrativa-, mientras que narraciones más complejas incluyen el resumen, la orientación, la

5. Se cuenta con la autorización del colaborador para compartir su información personal.

6. El glosado de datos fue revisado por especialistas de dos variantes del otomí: Ewald Hekking y Andrés de Jesús (1984), especialista del otomí de Santiago Mexquititlán, y María de Jesús Selene Hernández Gómez (HernándezGreen et al. 2011), especialista del otomí de Santa Ana Hueytlalpan, con quienes se realizó el consenso para el glosado del corpus propio.

7. Una cláusula es cualquier unidad que contenga un evento unificado, un predicado que exprese una sola actividad, situación, evento o estado. También se tomaron en cuenta las aportaciones de Aissen (2006) y

Palancar y Alarcón Neve (2007). De acuerdo con Aissen (2006), los elementos de una cláusula son como mínimo un predicado y sus argumentos, a lo que llama núcleo predicativo. Además, una cláusula incluye también: 1) una especificación de TAM; 2) una especificación de polaridad (afirmativo o negativo); 3) tal vez evidenciales, partículas deícticas, etc. Y, facultativamente: 4) adjuntos de varios tipos. Incluyen frases adverbiales/modificadores, un predicado secundario; $y$, 5) operadores (y las transformaciones que inducen; por ejemplo, el foco, la interrogación). Por su parte, Palancar y Alarcón Neve (2007) señalan que la cláusula contiene: 1) un solo sujeto, 2) un solo predicado principal, 3) una sola marca de TAM por predicado, 4) una sola marca de concordancia de sujeto por predicado, 5) una sola marca de polaridad por predicado, y 6) una unidad entonacional unificada (Palancar y Alarcón Neve 2007, 350).

8. De la propuesta de Labov (1972) se retoman los segmentos estructurales presentes en las narraciones. 
acción complicante, la resolución, la evaluación y la coda. En los siguientes apartados se presentan las estructuras prototípicas que se encontraron en los textos del corpus.

Con relación a las superestructuras de los textos, se encuentra que los dos temas analizados presentan dos distintas. En el caso de los textos que narran los eventos ocurridos durante el primer día en la ciudad, se trata de narraciones que presentan hechos inesperados que suscitan una complicación o ruptura de la cotidianeidad; mientras que los textos que describen los juegos de la infancia tienen una tendencia a ser representados con una estructura diferente que corresponde a lo que Schank y Abelson (1977) definen como guión, es decir, una estructura que evoca una secuencia estereotipada de acciones que definen una situación bien conocida o de eventos cotidianos. Esto quiere decir que, mientras que la narración cuenta con presentación de una complicación, el guión carece de este elemento. Las autoras señalan lo siguiente: que lo que se representa a partir de la experiencia en un guión es sólo una copia parcial de esa experiencia, que los guiones describen eventos cotidianos, y que son resultado del análisis de las representaciones de la experiencia. El guión tiene características similares a la narración, ya que es una estructura organizada temporalmente que posee secuencias de acción con una finalidad específica (Nelson 1996).

\subsubsection{Superestructura de las narraciones sobre experiencias personales en otomí}

De los 28 textos que conforman el corpus, 14 presentan la estructura propia de las narraciones ${ }^{9}$. Estas narraciones presentan unos mínimos comunes que corresponden a las categorías labovianas reconocidas como orientación, complicación y evaluación, es decir, presentan un elemento más con relación al mínimo estructural de la narración explicada por Labov (1972). Por su parte, el resumen no aparece en ninguna de las narraciones y la coda solamente se incluye en cuatro de ellas. Todos los demás elementos estructurales aparecen de manera variable en las diferentes narraciones analizadas en este estudio. En las siguientes secciones, se presentan los tipos de segmentos narrativos que aparecen en los textos y se describen las características más destacables de cada sección.

\subsubsection{Segmentos de orientación}

La función principal de los segmentos de orientación es brindar información contextual para ubicar a los personajes, la situación y el lugar en el que se desarrolla el relato. En las narraciones revisadas, los participantes pueden ser mencionados de manera explícita con nombres comunes o propios, o bien pueden ser expresados por medio de sufijos de persona o mediante el uso de proclíticos. En los ejemplos (1) y (2) se utiliza el enclítico enfático de primera persona de singular como sujeto $(=g a \mathrm{y}=k a)$, y en (3) el enclítico de primera persona plural exclusivo $=$ he para hacer referencia a los participantes del relato ${ }^{10}$. Además, el narrador hace uso de nombres comunes jwädä 'hermano' como en (2).
9. Diez de estos textos tratan sobre el primer día en la ciudad y cuatro sobre los juegos de infancia.
10. En esta investigación se usan las siguientes abreviaturas para los proclíticos, afijos y partículas del otomí de la colonia Nueva Realidad en la Ciudad de Querétaro: -: afijo; =: clítico; <>: morfema no concatenativo; 1 : primera persona; 2: segunda persona; 3 : tercera persona; A: aspecto; ACT: actualizador; APD: adjetivo usado como predicado; CCOD: cambio de código; D: forma dependiente; DAT: dativo; DEF: definido; DEIC: deíctico; DEM: demostrativo; DEP: dependiente; DET: determinado; DIM: diminutivo; DU: dual; END: endocéntrico; ENF: énfasis; EXCL: exclusivo; EXO: exocéntrico; FPREST: frase préstamo; IMP: imperfectivo; INCL: inclusivo; IND: indeterminado; INM: inmediativo; INTENS: intensificador; INTERJ: interjección; INTERR: interrogativo; IRR: irrealis; L: forma libre; LIM: limitativo; LOC: locativo; NEG: negativo; OBJ: objeto; PAS: pasado; PERF: perfectivo; PL: plural; PLU-PERF: plu-perfecto; POS: posesivo; PRED: predicativo; PRES: presente; PREST: préstamo; PROG: progresivo; RED: reducido; REL: relativo; REF: reformulación; REFL: reflexivo; RD: referencia distancia; $\mathrm{RH}$ : referencia hablante; RI: referencia interlocutor; SG: singular; TEMP: temporal. 
(1) pus dá ponga jam rantxo

$\begin{array}{llll}\text { pus } & d a ́=p o n=g a & j a=m & \text { rantxo } \\ \text { pues } & \text { 1.PAS=salir=1.ENF } & \text { LOC }=1 . P O S & \text { PREST } \\ \text { 'Pues me salí de mi rancho' (ciudad_9_2) } & \end{array}$

(2) ne bi zixkam jwädä

$\begin{array}{lll}\text { ne } \quad b i=z i x=k a=m & \text { jwädä } \\ \text { y } & \text { 3.PAS=traer }=1 . E N F=1 . P O S & \text { hermano } \\ \text { ' } Y \text { me trajo } & \text { mi hermano } a \text { mi' (ciudad_9_3). }\end{array}$
la traducción libre al español, se señala entre paréntesis el tópico al que corresponde cada texto (juegos o ciudad), seguido de un guión bajo se escribe el número de texto en el corpus; y, después de otro guión bajo, se señala el número de cláusula.

Con referencia a los locativos, estos pueden expresarse mediante sufijos de lugar al igual que el uso de nombres concretos. Véase en el ejemplo (3) que en una misma cláusula se especifica esta información por medio de un sufijo de locación (=wa) y un nombre propio (Monterrey). Dado el tema de la narración, se trata de las ciudades a las que llegan los hablantes en relación con el lugar de partida.

(3) dá ehewa Monterrey m'nagi

$$
\begin{array}{ll}
d a ́=e=h e=w a & \text { Monterrey m'nagi } \\
\text { 1.PAS=venir=PL.EXCL=LOC.RH } & \text { PREST } \\
\text { 'nos salimos para acá a Monterrey otra vez' (ciudad_9_17). }
\end{array}
$$

En general, las 14 narraciones presentan un uso frecuente de enclíticos de persona para indicar esta información orientativa más que la mención de los nombres comunes y propios de los diferentes participantes del relato. Sin embargo, sucede lo contrario con las referencias de lugar: es mayor la frecuencia de nombres propios y comunes que de sufijos y parece que la identificación de los participantes es menos relevante, ya que no se especifican mediante un sintagma nominal. La lengua no lo requiere, pues la información de persona está expresada en los enclíticos. En cambio, sí se especifican las referencias asociadas directamente con el tema de la narración - que, en este caso, son las ciudades-, como en (3) y (4), donde se menciona el nombre de la ciudad a la que los hablantes migraron.

(4) xtá ekawa jar nuwa jar nuwa Maxei

$\begin{array}{lll}x t a ́=e=k a=w a & j a=r & n u=w a \\ 1 . P E R F=v e n i r=1 . E N F=L O C . R H & L O C=S G & \mathrm{DEF}=\mathrm{LOC} . \mathrm{RH} \\ j a=r & n u=w a & \text { Maxei } \\ \mathrm{LOC}=\mathrm{SG} & \mathrm{DEF}=\mathrm{LOC} . \mathrm{RH} & \text { Querétaro } \\ \text { 'me he venido aquí aquí } a \text { Querétaro' (ciudad 1 } 15) . & \end{array}$

\subsubsection{Segmentos de complicación}

Los segmentos de complicación aparecen en todas las narraciones. Estos expresan el hecho más tenso de la historia, pues rompen la estabilidad que proporciona la orientación. Ponen de manifiesto los acontecimientos que permiten la ruptura de la cotidianeidad, es decir, presentan el hecho inesperado. La complicación es un componente esencial en el reconocimiento de una narrativa.

En el ejemplo (5) se expresa la complicación, en la que el participante cuenta que en cuanto llegó a la ciudad se perdió entre las calles, mientras intentaba regresar a la terminal de autobuses. La complicación aborda situaciones extremas que implican algún peligro para los hablantes, como extraviarse en la ciudad, no encontrar a los familiares o pasar la noche en las calles. 
(5) dá 'meka

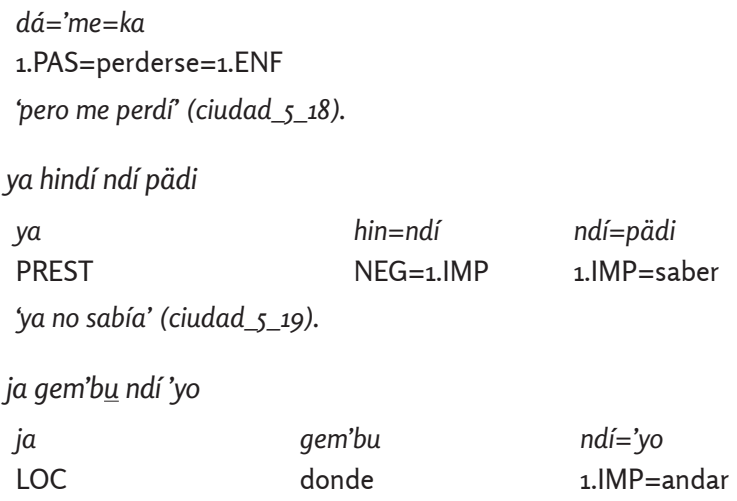

én dónde andaba' (ciudad_5_20).

\subsubsection{Segmentos de resolución}

Los segmentos de resolución aparecen en ocho de las narraciones sobre la ciudad. Este tipo de segmentos típicamente denota un evento o una serie de eventos posteriores a la complicación que permiten resolver o aclarar el conflicto presentado en el relato, como se ejemplifica en (6):

(6) dígem'bu, dá kwatuga 'nar ar nxumfo,

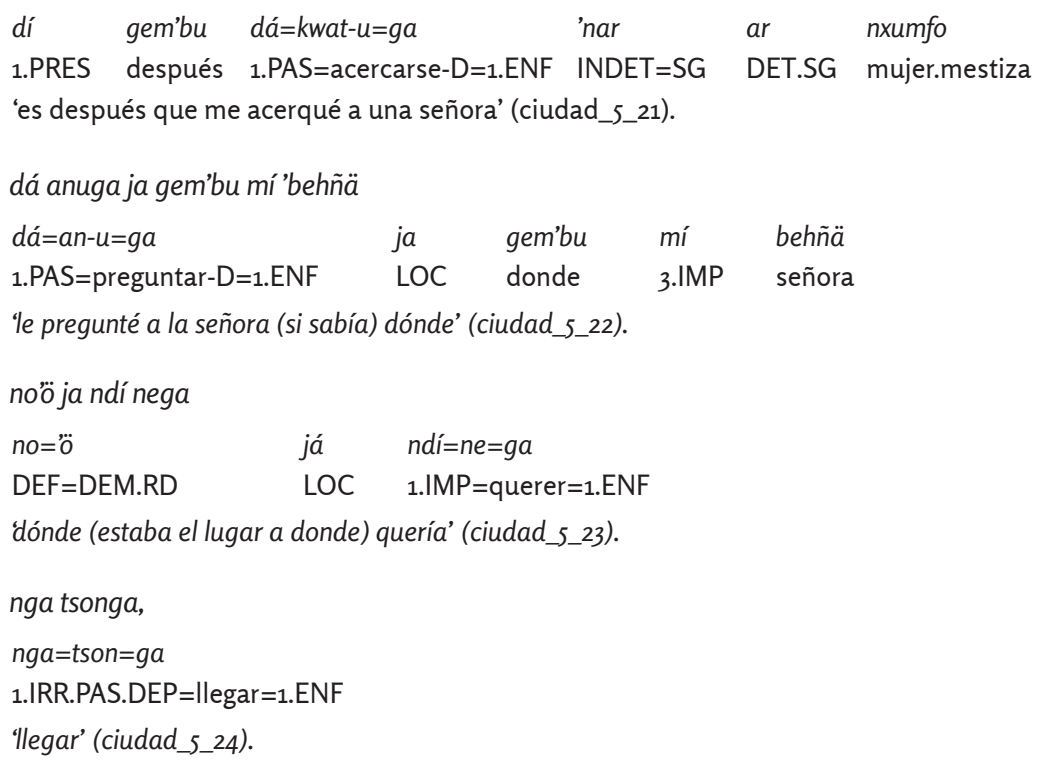

\subsubsection{Segmentos de evaluación}

$\mathrm{Al}$ igual que los segmentos de orientación y los de complicación, los de evaluación aparecen invariablemente en las narraciones analizadas. Se emplean como estrategia para marcar la actitud del narrador en relación con los sucesos presentados y los personajes involucrados en la historia. Marca la importancia de los elementos narrativos, es decir, por qué y para qué se narra (7). Por lo regular, las evaluaciones están asociadas con las situaciones de peligro dentro de la ciudad, lo que provoca el miedo y el deseo de regresar a su lugar de origen. 
(7) porke xí ndí tsu di gatho

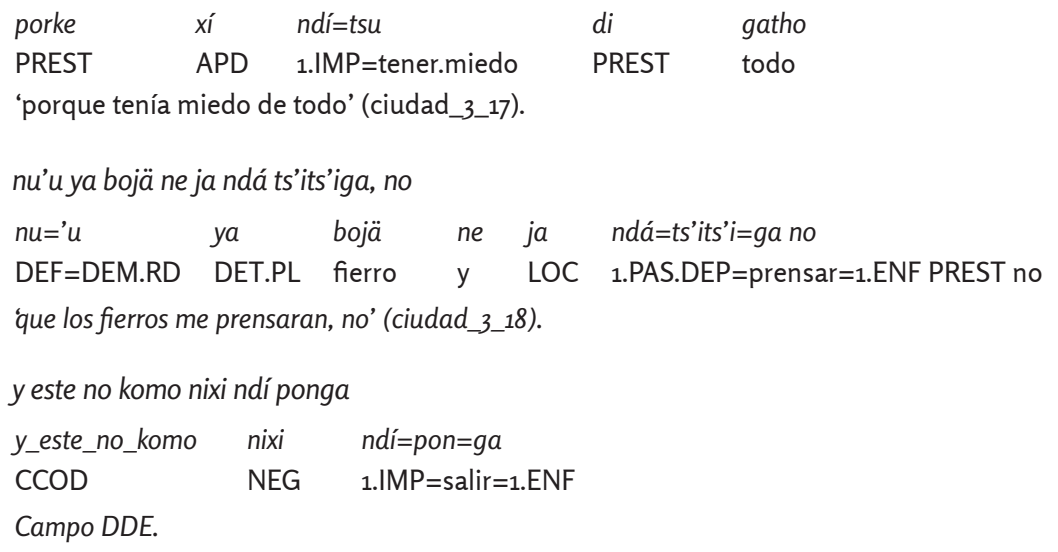

\subsubsection{Segmentos de coda}

Este tipo de segmentos narrativos permite regresar el discurso narrativo al momento presente de la enunciación y aparece como comentario final (8).

(8) ge bi nja’bu

\begin{tabular}{|c|c|c|}
\hline ge & $b i=n j a{ }^{\prime} b u$ & \\
\hline que & 3.PAS=ser.así & \\
\hline \multicolumn{3}{|c|}{ 'fue así' (ciudad_5_59). } \\
\hline \multicolumn{3}{|c|}{ nő̈ dá thoka nuwa } \\
\hline no $=\dddot{o}$ & $d a ́=t h o=k a$ & $n u=w a$ \\
\hline $\mathrm{DEF}=\mathrm{DEM} \cdot \mathrm{RD}$ & 1.PAS=llegar $=1 . \mathrm{ENF}$ & $\mathrm{DEF}=\mathrm{LOC} \cdot \mathrm{RH}$ \\
\hline \multicolumn{3}{|c|}{ 'lo que me pasó aqui” (ciudad_5_6o). } \\
\hline \multicolumn{3}{|l|}{ ja dá tsoka. } \\
\hline ja & dá $=\mathrm{tso}=\mathrm{ka}$ & \\
\hline LOC & 1.PAS=llegar $=1 . \mathrm{ENF}$ & \\
\hline
\end{tabular}

Los segmentos de coda aparecen en cinco de las narraciones del corpus. En uno de los textos se cierra con una cláusula que funciona narrativamente como coda y, al mismo tiempo, expresa una evaluación, pues ofrece un juicio de valor sobre la vida que lleva la narradora en la actualidad (9).

(9) pus ya dí 'buka xí hño'bya

$\begin{array}{lllll}\text { pus } & y a & d i ́=' b u=k a & x i ́ & h \tilde{o}=\text { 'bya } \\ \text { PREST } & \text { PREST } & \text { 1.PRES=vivir=1.ENF } & \text { INTENS } & \text { bien=ACT }\end{array}$

'pues ya vivo mejor ahora'.

\subsubsection{Superestructura de los guiones}

En este apartado se ejemplifican las características de los 14 textos estructurados como guiones ${ }^{12}$, siguiendo la propuesta de Schank y Abelson (1977). Estos autores señalan como componentes de los guiones: a) condiciones de entrada; b) accesorios; c) roles; d) resultado; e) caminos periféricos; $\mathrm{y}$, 12. De los 14 textos estructurados como guiones, 13 textos abordan la temática de juegos en la infancia y 1 sobre el primer día en la ciudad. f) escenas. Las condiciones de entrada son las conceptualizaciones o acciones que se deben cumplir antes para que el guión tenga lugar. Schank y Abelson (1987) indican que las condiciones de entrada se encuentran en un dominio macrocausal, en el sentido de que dichas condiciones, como su 
nombre lo indica, articulan al guión completo. Los accesorios son aquellos objetos que están involucrados en los eventos y las acciones se llevan a cabo gracias a la existencia de esos elementos. Hablar de roles es referirse a los participantes presentes en los eventos. Los resultados son las condiciones que deben ser ciertas después de que el guión se lleve a cabo e implican las consecuencias de los eventos. Por otro lado, puede haber variaciones en un guión. A estas divergencias Schank y Abelson (1977) las llaman caminos periféricos. Schank y Abelson (1987) agregan que los caminos periféricos en los guiones tienen lugar cuando la cadena de eventos causales no sigue su curso regular, esto por la existencia de estados o acciones que fungen como interferencias y distracciones. Por último, las escenas son la secuencia de eventos que ocurren. Estos son representados en una forma conceptual dependiente, en donde cada escena precede a otra, y para que una ocurra debe darse la acción anterior. Los guiones en otomí requieren de las condiciones de entrada, los accesorios y la mención de los roles como mínimos estructurales; la presencia de los elementos restantes es opcional.

\subsubsection{Las condiciones de entrada}

Los diferentes guiones presentan como marco del relato las labores que se desarrollan en el campo, es decir, el momento del juego en la mayoría de los relatos coincide con la actividad de cuidar animales. De modo que el juego ocurre dentro de una escena y un marco espacial y específicos. Esto constituye las condiciones de entrada, dado que el desarrollo del juego parece estar reservado a este marco general, como se observa en el ejemplo (10).

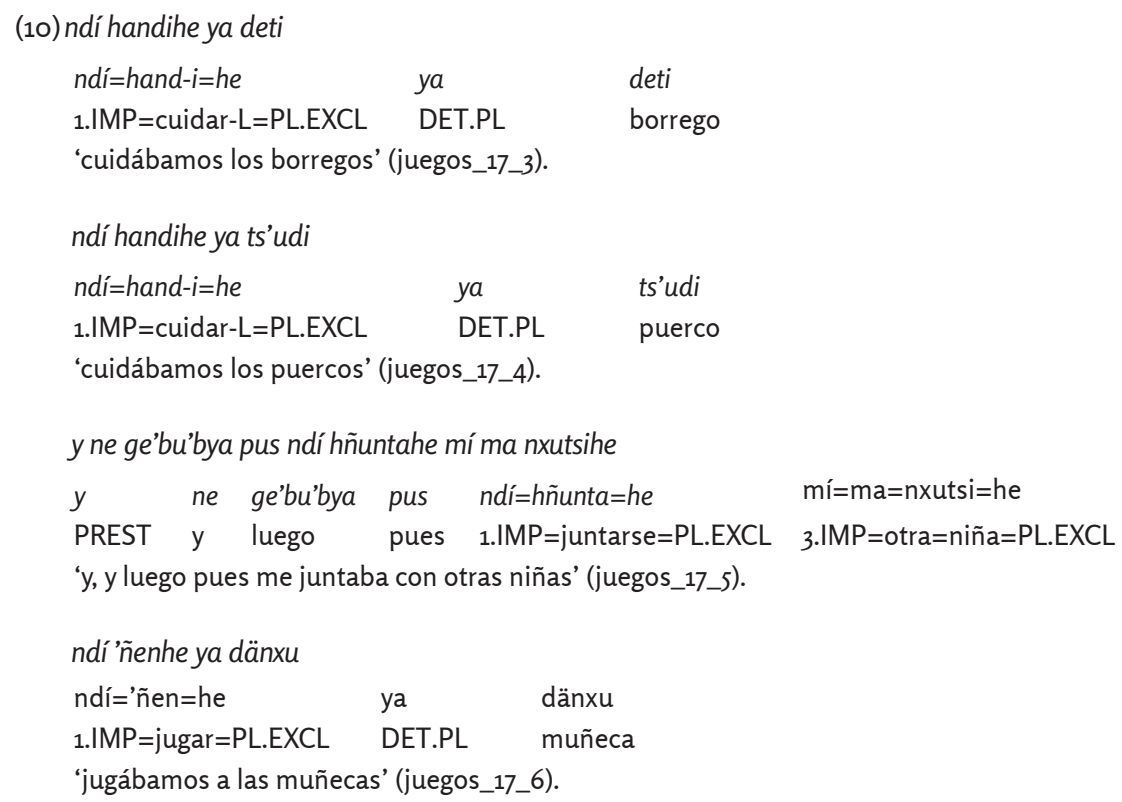

Además, para que los niños jueguen mientras cuidan a los borregos, caballos o puercos, es necesario que dichos animales cumplan con la condición de estar reunidos en un mismo sitio y a la vista de sus cuidadores. De lo contrario, el juego no tendría lugar, pues la actividad sería justamente recuperar los animales. 
La escena y marco espacial específicos en los relatos sobre los juegos de los otomíes tiene sentido si se entiende la dinámica social de este grupo indígena en su lugar de origen. Los otomíes desde niños ayudan a sus padres en el trabajo del campo, en la milpa, y colaboran con el cuidado de los animales. Los narradores de este estudio cuentan que, mientras participaban en estas labores, aprovechaban para jugar con otros niños. En los guiones sobre los juegos, los narradores otomíes representan el acontecer diario durante su infancia y se involucran en los eventos narrados como parte de ellos, como protagonistas y testigos de los hechos.

\subsubsection{Los accesorios}

El componente de los accesorios constituye uno de los elementos relevantes en los guiones de los otomíes. Los elementos presentes en el campo y la milpa se convierten en el material para que los niños otomíes fabriquen sus juguetes: muñecas, carritos y canicas hechos de lodo (11); flores (12), zacate, hojas de maíz, mazorcas, en algunos casos los animales funcionan como accesorios porque se les considera como juguetes (13). Dado que no contaban con juguetes, para divertirse hacían uso de su ingenio y de los elementos de la naturaleza que se encontraban a su alcance para elaborar sus juguetes. El hecho de que en los guiones otomíes se compartan las escenas, marco espacial y los accesorios con los que los niños se divierten en la comunidad, subraya la identidad cultural de los miembros de este grupo originario.

(11) ga xa'he ya bohai

$$
\begin{array}{lll}
g a=x a \text { '=he } & y a & \text { bo_hai } \\
\text { 1.IRR.PRES=rascar=PL.EXCL } & \text { DET.PL } & \text { negro_tierra } \\
\text { 'arrancaremos el lodo' (juegos_16_46). } &
\end{array}
$$

(12) díxat'ihe ya doni

$$
\begin{array}{lll}
d i=x a t '-i=h e & y a & \text { doni } \\
\text { 1.PRES=deshierbar-L=PL.EXCL } & \text { DET.PL } & \text { flor } \\
\text { 'cortamos las flores' (juegos_16_51). } & &
\end{array}
$$

(13) ga japuga yoho ya txuku

$\begin{array}{llll}g a=j a p-u-g a & \text { yoho ya } & \text { txuku } \\ \text { 1.IRR.PRES=hacer-D=1.ENF dos DET.PL } & \text { perro } \\ \text { 'lo haré con los dos perros' (juegos_16_58) } & \end{array}$

\subsubsection{Los roles}

Los roles corresponden a los participantes presentes en los relatos y la mención de estos, como es de esperarse, se da en todos los guiones de los otomíes. Como en el caso de las narraciones, en los guiones se presenta a los participantes a través de enclíticos de sujeto, como en el ejemplo (14), en el que se usa el enclítico enfático de primera persona del singular como sujeto $=g a$ ' 1 .ENF'. En este caso, se busca privilegiar el punto de vista del narrador como protagonista de los eventos narrados y como testigo de las acciones realizadas por otros participantes en el relato. 
(14) ga japugawa,

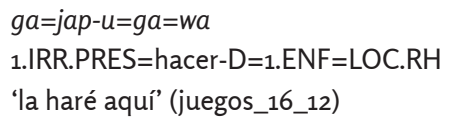

En el ejemplo (15) se presenta a los participantes con el enclítico =he '1.PL. EXCL' (primera persona del plural que excluye al interlocutor). Aunque en el guión no se manifiesta de manera explícita quiénes son estos participantes, se infiere que quien relata la historia juega con otras niñas.

(15) dí hokihe má lele

$\begin{array}{lll}\text { dí=hok-i=he } & \text { má } & \text { lele } \\ \text { 1.PRES=hacer-L=PL.EXCL } & 1 . P O S & \text { bebé } \\ \text { 'hacemos nuestras muñecas' (juegos_16_56) } & \end{array}$

\subsubsection{Los resultados}

Los resultados son las consecuencias de los eventos, las condiciones que deben ser ciertas después de que los eventos en el guión se lleven a cabo. Este componente aparece en siete de los catorce guiones analizados. Un resultado en este tipo de guiones se encuentra cuando después de elaborar las muñecas se abre la posibilidad del juego. En (16) se presenta un diálogo en el que una niña habla con otras participantes y les pregunta si ya terminaron de hacer sus muñecas para jugar con ellas:

(16) ya gem'bya xtá wadi

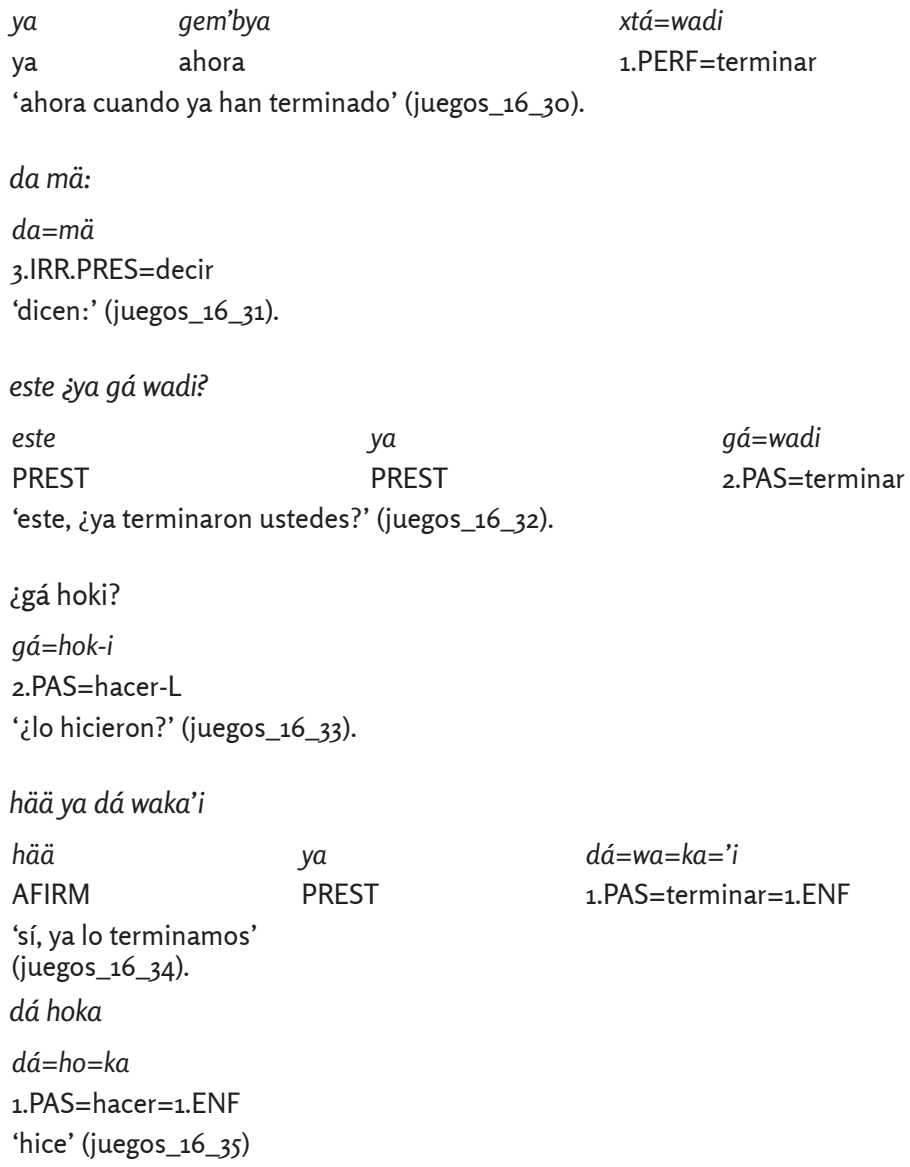


äää weno hange nu ma 'ñenu bätsihu

äää weno hange nu ma='ñen- $u$ bätsi=hu

ah bueno entonces TEMP INM=jugar-D niño=PL.INCL

‘jah, bueno! entonces vamos a jugar’ (juegos_16_36).

\subsubsection{Las escenas}

Las escenas corresponden con la secuencia de eventos que ocurren en el guión y son representados en una forma dependiente, en donde cada escena precede a otra. En el ejemplo (17) se muestra un extracto de un guión en el que se mencionan varias escenas que ocurren de manera secuencial. Las niñas deben hacer primero los collares para enseguida ponérselos a sus caballos y después montarlos. En general, en las escenas narradas por los otomíes se manifiestan aspectos culturales que son compartidos entre los miembros de esta comunidad, de manera que los guiones asumen el punto de vista de los narradores y representan la identidad de este grupo originario.

(17) ndí hokuhe ya thebe

\begin{tabular}{|c|c|}
\hline ndí=hok-u=he & $y a$ \\
\hline 1.IMP=hacer-D=PL.EXCL & DET.PL \\
\hline \multicolumn{2}{|c|}{ ‘hacíamos los collares’ (juegos_14_3). } \\
\hline \multicolumn{2}{|l|}{ ndí tutuhe mfani } \\
\hline ndí=tut-u=he & $m=$ fani \\
\hline 1.IMP=colgar $-\mathrm{D}=\mathrm{PL} . \mathrm{EXCL}$ & 1.POS=caballo \\
\hline \multicolumn{2}{|c|}{ ‘se los colgábamos a nuestro caballo’ (juegos_14_4). } \\
\hline \multicolumn{2}{|l|}{ ne ndí ntoge mfani, } \\
\hline ndí=ntoge & $m=$ fani \\
\hline 1.IMP=montar & 1.POS=caballo \\
\hline
\end{tabular}

\section{Conclusiones}

En este trabajo se describieron los textos narrativos sobre experiencias personales en hablantes del otomí de Santiago Mexquititlán. Se encontró que el tópico condiciona en buena medida el tipo de estructura textual. Cuando se habla sobre el primer día en la ciudad, se utilizan narraciones que configuran eventos inesperados que rompen con la cotidianeidad; mientras que los textos que describen los juegos de la infancia tienen una tendencia a ser representados a modo de guión, es decir, a manera de descripción habitual.

Se identificaron mínimos estructurales para que una narración o un guión en otomí se lleve a cabo. En el caso de las narraciones se debe cumplir con tres componentes; se trata de la orientación, la complicación, y la evaluación. Los primeros dos coinciden con los elementos mínimos en la narración propuestos por Labov (1972), y se suma la evaluación en las narraciones otomíes.

En la orientación, los hablantes ofrecen información referencial sobre los lugares, justamente, porque son los escenarios desconocidos, que se constituyen en el tema de la narración en los textos asociados con la migración; mientras que los participantes en los relatos son nombrados por medio de 
enclíticos de plural y enclíticos enfáticos de sujeto. Por su parte, la complicación, se liga directamente a los riesgos que corren los hablantes en una ciudad desconocida, sin familia, sin empleo y sin un lugar al cual llegar. Y en las evaluaciones los narradores emiten los sentimientos que afloraban cuando enfrentaban dichas dificultades.

En el caso de los guiones, se privilegian las condiciones de entrada, los accesorios y los roles. La situación marco o condición de entrada que condiciona el relato es el cuidado de los animales en la milpa o en el campo. En relación con los accesorios, involucra los elementos necesarios para que la acción habitual se lleve a cabo. Se trata de lodo, flores, zacate, hojas de maíz, mazorcas, entre otros, que dan lugar a los juguetes utilizados y, por último, los roles que identifican a los actores de los relatos. En este sentido, los hablantes construyen discursivamente una niñez en el campo que focaliza el espacio y los recursos que ofrece el entorno.

De igual manera, se muestra que los textos sobre experiencias personales pertenecientes a los hablantes del otomí pueden ser descritos según las clasificaciones realizadas para otras lenguas, lo que sugiere que la estructura de las narraciones o guiones no parece estar condicionada por el tipo de lengua, de modo que la disposición de los elementos estructurales en el texto se encuentra motivada por aspectos sociales y culturales que viven los hablantes y que se articulan según su intención comunicativa.

Por último, es importante insistir en la necesidad de recopilar historias sobre experiencias personales en las lenguas originarias y realizar estudios desde una perspectiva discursiva, de manera tal que se contribuya con la revitalización y mantenimiento de las lenguas descritas y, por ende, de la cultura de una comunidad. 


\section{Q Bibliografía}

" Aissen, Judith. 2006. Taller sobre la complementación. México: Centro de Investigaciones y de Estudios Superiores en Antropología Social-Sureste, 31 de julio al 10 de agosto de 2006.

" Bamberg, Michael. 1997. "A constructivist approach to narrative development". En Narrative development: Six approaches, editado por Michael Bamberg, 85-132. Mahwah: Lawrence Erlbaum.

» Bartholomew, Doris. A. 1984. "Algunas diferencias entre la narración oral y la narración escrita, con referencia a una muestra en el otomí del Estado de México". SIL Mexico Workpaper, 5:52-62.

" Bartholomew, Doris. 1995. "Difrasismo en la narración otomí". En Vitalidad e influencia de las lenguas indígenas, editado por Ramón Arzápalo y Yolanda Lastra, 449-463. Ciudad de Mexico: Universidad Autónoma de Querétaro.

" Berman, Ruth y Dan Slobin. 1994. Relating events in narrative: A crosslinguistic developmental study. New Jersey: Lawrence Erlbaum.

"Boersma, Paul y David Weenink. 1992. "Praat: doing phonetics by computer". Amsterdam: Universidad de Amsterdam. http://www.fon.hum.uva.nl/praat/.

" Bruner, Jerome. 1986. Actual minds, possible worlds. Cambridge, MA: Harvard University Press.

" Castillo, Carolina. 2014. "Observaciones sobre la interfaz sintaxis-pragmática en narrativas de tres lenguas indígenas mexicanas". Signo y Seña 25: 35-58.

»Comrie, Bernard y Martin Haspelmath. 2015. The Leipzig glossing rules: Conventions for interlinear morpheme-by-morpheme glosses. Leipzig: University of Leipzig. https://www. eva.mpg.de/lingua/resources/glossing-rules.php.

»Donati, Pierpaolo. 1999. La ciudadanía societaria. Granada: Editorial Universidad de Granada.

》 Hekking, Ewald y Severiano Andrés de Jesús. 1984. Gramática otomí. Santiago de Querétaro: Universidad Autónoma de Querétaro.

" Hekking, Ewald, Severiano Andrés de Jesús, Paula de Santiago Quintanar, Alonso Guerrero y Roberto Aurelio Núñez. 2010. He’mi Mpomuhñä ar Hñäñho ar Hñämfo Ndämaxei / Diccionario bilingüe otomí-español del estado del Querétaro. México, D.F: Instituto Nacional de Lenguas Indígenas (INALI).

" Hernández-Green, Nestor, Enrique Palancar y Selene Hernández Gómez. 2011. The Spanish loanword ladoin Otomi spatial descriptions. Language Sciences 33.6: 961-980.

»Labov, William. 1972. Sociolinguistic patterns. Philadelphia: University of Pennsylvania Press.

" Labov, William y Joshua Waletzky. 1997 [1967]. “Narrative Analysis: Oral version of Personal Experience". Journal of Narrative and Life History 7: 1-4.

" Li, Pin, Fan Zhang, Erlfang Tsai y Brendan Puls. 2014. "Language history questionnaire (LHQ 2.0): A new dynamic web-based research tool”. Bilingualism: Language and Cognition 17.3: 673-680.

" McCabe, Allyssa. 1997. "Developmental and cross-cultural aspects of children's narrations”. En Narrative development: Six approaches, editado por Michael Bamberg, 137-174. Mahwah, NJ: Lawrence Erlbaum Associates. 
» Nava López, Enrique Fernando. 2010. “El catálogo de las lenguas indígenas nacionales y la lingüística otopame." En Homenaje a Yolanda Lastra: X Coloquio Internacional sobre otopames, editado por Ana María Salazar y Verónica Kugel, 81-122. Ciudad de México: Universidad Nacional Autónoma de México, Instituto Nacional de Lenguas Indígenas México.

» Nelson, Katherine. 1996. Language in cognitive development. Nueva York: Cambridge University Press.

» Nicolopoulou, Agelike. 1997. “Children and narratives: Toward an interpretive and sociocultural approach”. En Narrative development: Six approaches, editado por Michael Bamberg, 174-216. Mahwah: Lawrence Erlbaum.

» Ochs, Elinor. 1992. “Indexing gender”. En Rethinking context: Language as an interactive phenomenon, editado por Alessandro Duranti y Charles Goodwin, 335-358. Cambridge: Cambridge University Press.

» Ochs, Elinor. 1997. “Narrative”. En Discourse as structure and process, editado por Teun van Dijk, 185-207. Londres: SAGE Publications.

»Palancar, Enrique. 2009a. Gramática y textos del hñöñhö: Otomí de San Ildefonso Tultepec, Querétaro. México: Universidad Autónoma de Querétaro, Plaza y Valdés.

»Palancar, Enrique. 2009b. “Nor Kamalion. El Camaleón”. Tlalocan 16: 51-84.

"Palancar, Enrique y Luisa J. Alarcón Neve. 2007. “Predicación secundaria depictiva en español”. Revista Española de Lingüística 37: 337-370.

» Quasthoff, Uta. 1997. “An interactive approach to narrative development”. En Narrative Development: Six Approaches, editado por Michael Bamberg, 45-84. Mahwah: Lawrence Erlbaum.

" Rico, Karola. 2014. “Dí pengi ga pot’i, Volverme a sembrar: Propuesta didáctica para la enseñanza de la lengua y la cultura ñäñho en la colonia Nueva Realidad". Tesis de Maestría. Santiago de Querétaro: Universidad Autónoma de Querétaro.

»Schank, Roger y Robert P. Abelson. 1977. Scripts, plans, goals, and understanding. Hillsdale, Nueva Jersey: Lawrence Erlbaum.

»Schank, Roger y Robert P. 1987. Guiones, planes, metas y entendimiento. Barcelona: Paidós.

»Stein, Nancy y Elizabeth Albro. 1997. “Building complexity and coherence: children's use of goal-structured knowledge in telling stories". En Narrative development: Six approaches, editado por Michael Bamberg, 5-44. Mahwah: Lawrence Erlbaum.

» Thornborrow, Joanna. 2012. "Narrative analysis”. En The Routledge handbook of discourse analysis, editado por Paul Gee y Michael Handford, 51-65. Nueva York: Routledge.

» Thornborrow, Joanna y Jennifer Coates. 2005. The sociolinguistics of narrative. Amsterdam: John Benjamins.

»Van Dijk, Teun A. 1978. La ciencia del texto. Un enfoque interdisciplinario. Barcelona: Paidós.

»Van Dijk, Teun A. 1980. Macrostructures. An Interdisciplinary Study of Global Structures in Discourse, Interaction and Cognition. Hillsdale, Nueva Jersey: Lawrence Erlbaum.

»Van Valin, Robert D. 1999. "A typology of the interaction of focus structure and syntax". En Typology and the theory of language: From description to explanation, editado por Ekaterina V. Rakhilina y Yakov G. Testelec. Moscú: Languages of Russian Culture. 